\title{
ATENÇÃO EM SAÚDE NO CICLO GRAVÍDICO-PUERPERAL: REVISÃO INTEGRATIVA DE LITERATURA
}

\author{
Nathalia Cristina Plaza Caprioli ${ }^{1}$, Kátia Terezinha Alves Rezende ${ }^{2}$ Cassia Regina Fernandes \\ Biffe Peres ${ }^{3}$,Silvia Franco da Rocha Tonhom ${ }^{4}$, Aline Pererira de Souza ${ }^{5}$ \\ ${ }^{1}$ Secretaria Municipal de Saúde de Marília, São Paulo, Brasil, nathaliaplazabasta@hotmail.com \\ ${ }^{2,3,4 \text { e } 5}$ Faculdade de Medicina de Marilia (Famema), Brasil, ${ }^{2}$ katialvesrezende@gmail.com; ${ }^{3}$ c.r.biffe@gmail.com \\ ${ }^{4}$ siltonhom@gmail.com, ${ }^{5}$ aline.psouza_enfer@hotmail.com
}

\begin{abstract}
Resumo: Objetivo: analisar a produção bibliográfica acerca da atenção à em saúde no ciclo gravídico puerperal na perspectiva das mulheres e da avaliação das ações pelos serviços. Método: Trata-se de uma pesquisa qualitativa por meio da Revisão Integrativa de Literatura. Utilizou-se as bases de dados BDENF, LILACS e Index Psicologia, por meio dos descritores "cuidado pré-natal", "período pós-parto", "serviços de saúde materno-infantil", "cuidados de enfermagem", "assistência médica" e "assistência integral à saúde". Elegeu-se 24 artigos para compor o corpus da análise. Resultados: Apresenta-se as temáticas, quais sejam, ações desenvolvidas pela equipe de saúde no ciclo gravídico puerperal; educação em saúde no ciclo gravídico puerperal; processo de trabalho da equipe e critérios para avaliação do cuidado no ciclo gravídico-puerperal. Conclusão: Depreende-se que muitos avanços ainda são necessários para que a atenção no ciclo gravídico puerperal seja orientada de acordo com seus pressupostos.
\end{abstract}

Palavras Chave: Cuidado Pré-Natal; Período Pós-Parto: Serviços de Saúde Materno Infantil;Cuidados de Enfermagem; Assistência Médica; Assistência Integral à Saúde.

\section{HEALTH CARE IN THE PREGNANCY-PUERPERAL CYCLE: INTEGRATIVE LITERATURE} REVIEW FOR THE

\begin{abstract}
Objective: to analyze the bibliographic production about health care in the puerperal pregnancy cycle from the perspective of women and the evaluation of actions by services. Method: This is a qualitative research through the Integrative Literature Review. The databases BDENF, LILACS and Index Psicologia were used, using the keywords "prenatal care", "postpartum period", "maternal and child health services", "nursing care", "medical assistance" And" comprehensive health care ". 24 articles were chosen to compose the corpus of the analysis. Results: The themes are presented, namely, actions developed by the health team in the puerperal pregnancy cycle; health education in the postpartum pregnancy cycle; team work process and criteria for evaluating care in the pregnancy-puerperal cycle. Conclusion: It appears that many advances are still needed so that the attention in the puerperal pregnancy cycle is oriented according to its assumptions.
\end{abstract}

Keywords: Pre-natal care; Postpartum period; maternal and child health services; Nursing care; medical assistance and Comprehensive health care.

\section{INTRODUÇÃO}

A Rede Cegonha é uma estratégia do Ministério da Saúde, operacionalizada pelo SUS, fundamentada nos princípios da humanização e assistência, onde mulheres, recémnascidos e crianças têm direito a: ampliação do acesso, acolhimento e melhoria da qualidade do pré-natal; Transporte tanto para o pré-natal quanto para o parto; vinculação da gestante à unidade de referência para assistência ao parto; realização de parto e nascimento seguros, através de boas práticas de atenção; acompanhante no parto, de livre 
escolha da gestante; Atenção à saúde da criança de 0 a 24 meses, e acesso ao planejamento reprodutivo. Seus principais objetivos são: um novo modelo de atenção ao parto, ao nascimento e à saúde da criança; uma rede de atenção que garanta acesso, acolhimento e resolução das adversidades; e redução da mortalidade materna e neonatal (Brasil, 2011). A Rede Cegonha tem como uma de suas estratégias o apoio à qualificação do cuidado em maternidades prioritárias, que tem produzido acúmulo de metodologias, experiências e conhecimento, bem como evidencia a necessidade de se atuar também na formação dos novos profissionais, a partir da prática. A identificação dessa necessidade culminou na formulação do projeto ApiceOn, que aponta para um processo de mudanças, ou de afirmação de um modelo que traz em sua base estruturante a integração de três dimensões bem definidas e inseparáveis: formação, atenção e gestão (Brasil, 2017). Luz, Aquino e Medina (2018) investigaram características da estrutura das unidades de saúde e dos processos gerenciais e assistenciais da Atenção Pré-Natal (APN) no âmbito da Atenção Primária à Saúde (APS) no Brasil, em municípios que aderiram ao Programa Nacional de Melhoria do Acesso e da Qualidade da Atenção Básica (PMAQ-AB) e constataram que a estrutura das unidades básicas e as ações prestadas pelas equipes não obedeciam à maioria dos padrões, destacando-se a existência de barreiras estruturais, indisponibilidade de medicamentos e exames essenciais, problemas na oferta do elenco de ações assistenciais, envolvendo a atenção individual e o cuidado clínico, bem como de promoção da saúde e ações coletivas e domiciliares ofertadas. Dessa forma, concluíram-se que, apesar da alta cobertura da APN e da sua institucionalização nos serviços de APS, persistem problemas que devem ser alvo de iniciativas governamentais que garantam atenção integral e de qualidade no ciclo gravídico-puerperal e que repercutam na melhoria dos indicadores de saúde materno-infantil Luz, Aquino e Medina (2018).

Spindola et al, 2017 na perspectiva de conhecer a vivência das mulheres no pós-parto e discutir a contribuição da enfermagem para educação, concluíram que o pós-parto é um período singular na vida da mulher. Evidenciaram sentimentos e sensações boas, mudanças no cotidiano, participação dos familiares nos cuidados com o bebê e realização de atividades de educação em saúde e que o enfermeiro contribui nessas ações com as mulheres durante o pré-natal, nas consultas de enfermagem ou nas orientações coletivas, entretanto não foram percebidas o seu desenvolvimento no puerpério.

Identificou-se que os serviços da APS do município estão com dificuldades em realizar ações individuais e ou coletivas às mulheres no ciclo gravídico-puerperal preconizadas pelas 
políticas de saúde. Assim, questiona-se: De que forma os serviços de saúde da APS estão realizando ações individuais e ou coletivas às mulheres no ciclo gravídico puerperal?

\section{OBJETIVO}

Analisar a produção bibliográfica acerca da atenção à saúde no ciclo gravídico-puerperal na perspectiva das mulheres e da avaliação das ações pelos serviços.

\section{MÉTODO}

Trata-se de uma pesquisa qualitativa, na qual a Revisão Integrativa de Literatura (RIL) foi eleita para alcance do objetivo delineado neste estudo. A Revisão Integrativa de Literatura é realizada por meio de uma análise ampliada de produções bibliográficas sobre um determinado campo do conhecimento, cujo objetivo principal é a obtenção de novas informações e significados para melhor compreensão de um fenômeno (Souza, Silva \& Carvalho, 2010). Na perspectiva do rigor metodológico da Revisão Integrativa da Literatura seguiram-se as seguintes etapas (Ganong, 1987; Whittemore \& Knafl, 2005).

Na primeira, identificou-se o problema, com definição do tema e a questão de pesquisa. $\mathrm{Na}$ segunda etapa, a seleção da amostra deu-se pela busca nas bases de dados BDENF, LILACS e Index Psicologia, por meio dos descritores "cuidado pré-natal", "período pósparto", "serviços de saúde materno-infantil", "cuidados de enfermagem", "assistência médica" e "assistência integral à saúde", os quais foram integrados por meio do operador "and" e "or".

Foram incluídos na revisão artigos completos relacionados ao objeto de pesquisa, publicados em português, originados de periódicos nacionais e internacionais, indexados nas bases de dados referidas, no período de janeiro de 2013 a junho de 2018 .

Foram excluídos artigos do tipo revisão e comentário, bem como aqueles que se relacionavam-se ao tema, porém com outro enfoque. Na terceira etapa, identificou-se 139 artigos, desses $16(11,5 \%)$ discorriam sobre a temática, cuidado em saúde no pré-natal e puerpério, porém $13(9,3 \%)$ tratavam de condições clínicas específicas da gestante, sendo elas, dependência química, risco para pré-eclâmpsia, soropositivo para HIV (Vírus da Imunodeficiência Adquirida), cardiopatias, obesidade, depressão e três (2,1\%) de situação social específica, estar encarceradas. Assim, esses foram excluídos pela leitura do título da investigação, permanecendo-se com 123 artigos. Esses foram submetidos a um processo 
de seleção considerando o título e o resumo. Conclui-se que 83 artigos (59,7\%) não discorriam sobre a temática em questão e sete $(5,0 \%)$ era estudo de revisão sistemática, sendo estes excluídos. Assim, selecionou-se 33 (23,7\%) artigos para leitura na íntegra. Desses, excluiu-se nove $(27,3 \%)$, foram excluídos, pois não respondiam à questão da pesquisa. Portanto, elegeu-se 24 (72,7\%) artigos para compor o corpus da análise.

$\mathrm{Na}$ quarta etapa procedeu-se a análise dos resultados, identificando-se convergências e divergências. E por fim, esses são apresentados e discutidos, por meio de quatro temáticas que emergiram, quais sejam, ações desenvolvidas pela equipe de saúde no ciclo gravídico puerperal; educação em saúde no ciclo gravídico puerperal; processo de trabalho da equipe e critérios para avaliação do cuidado no ciclo gravídico-puerperal.

\section{RESULTADOS}

\subsection{Caracterização dos artigos}

Caracterizou-se os artigos quanto ao ano de publicação, periódico, formação acadêmica dos autores, tipo de estudo e local de estudo. Quanto ao ano de publicação, identifica-se que seis $(25 \%)$ artigos foram publicados em 2015 , cinco $(20,8 \%)$ em 2016 , quatro $(16,7 \%)$ em 2013, outros quatro (16,7\%) em 2014, outros quatro (16,7\%) em 2017 e um (4,1\%) em 2018.

Em relação ao periódico, encontra-se que 15 (62,5\%) artigos foram publicados em periódicos de enfermagem, sete $(29,1 \%)$ em saúde coletiva, um $(4,2 \%)$ em medicina e um $(4,2 \%)$ em ciências da saúde. No quesito formação acadêmica dos autores, verifica-se que dez $(41,8 \%)$ correspondem a enfermagem, um $(4,1 \%)$ nutrição e enfermagem, um $(4,1 \%)$ medicina e enfermagem, um (4,1\%) fisioterapia e enfermagem, um $(4,1 \%)$ biomedicina e enfermagem, um $(4,1 \%)$ da nutrição e nove $(37,7 \%)$ dos artigos não constam a formação acadêmica.

O tipo de estudo representa-se por sete $(29,2 \%)$ artigos com abordagem quantitativa, nove $(37,5 \%)$ qualitativa e oito (33,3\%) não explicita sua abordagem. Quanto ao local de estudo, observa-se que $14(58,4 \%)$ foram realizados no nível de atenção primária, um $(4,1 \%)$ primário e secundário, seis (25\%) nível terciário e três (12,5\%) não registraram o local de estudo. 
Quadro 1 - Apresentação dos temas e resultados

\begin{tabular}{|c|c|}
\hline Temas & Resultados \\
\hline $\begin{array}{c}\text { Ações desenvolvidas } \\
\text { pela equipe de saúde } \\
\text { no ciclo gravídico } \\
\text { puerperal }\end{array}$ & $\begin{array}{l}\text { Apoio da equipe para amamentação (Barbosa et al, 2018). Descrevem as ações } \\
\text { pré-natal e puerpério realizadas tanto individual e coletivo no projeto. (Valdes, } \\
\text { Santos \& Prado, 2017). Consultas prepararam para serem mães. (Demarchi et al, } \\
\text { 2017). Frustrações por não terem consulta de enfermagem no pré-natal e puerpério. } \\
\text { Demarchl et al, 2017). Ações enfermeira e ACS em VD no puerpério (Corrêa et al, } \\
\text { 2017). Visita profissional equipe no primeiro mês durante puerpério; não receberam } \\
\text { visita pela equipe (Corrêa et al, 2017).O foco do atendimento do puerpério é na } \\
\text { criança (Corrêa et al, 2017). Orientações dos ACS são insuficientes diante das } \\
\text { necessidades das puérperas (Corrêa et al, 2017). Anticoncepção abordada na } \\
\text { puericultura (Corrêa et al, 2017). Não realizou cuidado as necessidades da puérpera } \\
\text { (Corrêa et al, 2017).Descreve as ações no período pré-natal, parto e pós parto } \\
\text { (Pedraza, 2016).Descrito ações realizadas no acompanhamento pré - natal (Santos } \\
\text { et al, 2016). }\end{array}$ \\
\hline $\begin{array}{l}\text { Educação em saúde no } \\
\text { ciclo gravídico } \\
\text { puerperal }\end{array}$ & $\begin{array}{l}\text { Orientações nutricionais (Barbosa, et al, 2018). Orientação aleitamento materno } \\
\text { pela equipe (Barbosa et al, 2018). Lacuna orientações ao aleitamento materno } \\
\text { (Tamara et al, 2017). Orientação de aleitamento realizada pelo médico (rodrigues et } \\
\text { al, 2016). Orientação não foi dada a todas as mulheres (Rodrigues et al, 2016). } \\
\text { Adolescentes grávidas recebem orientações relativas ao parto, da equipe e, em } \\
\text { especial do enfermeiro (Monteiro \& Oliveira et al, 2016). Fragilidade na adesão aos } \\
\text { grupos de gestantes (Monteiro \& Oliveira et al, 2016). Importância da participação } \\
\text { do grupo de gestante (Monteiro \& Oliveira et al, 2016). Recebeu orientação no pré- } \\
\text { natal (Barbosa et al, 2015). mulheres com mais estudo e as primíparas receberam } \\
\text { mais orientações sobre aleitamento materno (Barbosa et al, 2015).Temas } \\
\text { abordados pelo enfermeiro sobre orientações no pré-natal, parto e puerpério } \\
\text { (Oliveira et al, 2015). Primíparas necessita de mais orientações que multíparas } \\
\text { (Oliveira et al, 2015). Receberam orientações e sanaram dúvidas sobre aleitamento } \\
\text { materno no pré-natal e puerpério (Aparecida et al, 2014).Grupo sobre aleitamento } \\
\text { materno exclusivo; algumas tiveram clareza sobre o período e outras não } \\
\text { (Aparecida et al, 2014).Em grupo foi abordado sobre utilização de líquidos, } \\
\text { mamadeiras e chupetas; introdução precoce da chupeta (Aparecida et al, 2014). } \\
\text { Orientação sobre AM no pré-natal e no hospital, de forma individual e consideradas } \\
\text { insuficientes (Silva et al, 2014). Educação aprendida pelos pais; educação } \\
\text { verticalizada tanto na escola como nos serviços de saúde (Guerreiro et al, 2014). } \\
\text { Educação realizada individualmente retirando a oportunidade da construção coletiva } \\
\text { do conhecimento (Guerreiro et al, 2014).Primíparas reconhecem a família no } \\
\text { processo educativo e as multíparas consideram o profissional médico (Rodrigues et } \\
\text { al, 2013).Informações no pré-natal com foco no papel de mãe e na saúde do bebê } \\
\text { em detrimento a saúde da mulher (Cabral, Hirt \& Sand, 2013). Rede familiar e social } \\
\text { como apoio ao cuidado (Cabral, Hirt \& Sand, 2013).Dificuldade amamentação } \\
\text { (Corrêa et al, 2017). }\end{array}$ \\
\hline $\begin{array}{c}\text { Processo de trabalho } \\
\text { da equipe }\end{array}$ & $\begin{array}{l}\text { Queixa da irregularidade de visitas do ACS no puerpério (Corrêa et al, 2017).A } \\
\text { equipe não fornece consulta pós-parto (Corrêa et al, 2017).Fragilidade no } \\
\text { agendamento da consulta médica puerperal (Corrêa et al, 2017).Problema } \\
\text { organização no serviço para o agendamento (Corrêa et al, 2017). Aprovou } \\
\text { atendimento da recepção por demanda na amamentação (Corrêa et al, } \\
\text { 2017). Desejo de iniciativa da equipe para questionamento e esclarecer dúvidas } \\
\text { (Corrêa et al, 2017). Algumas dúvidas/ necessidades não são manifestas pelas } \\
\text { puérperas (Corrêa et al, 2017). Acesso ao agendamento como facilitador do pré- } \\
\text { natal (Cabral, Hirt \& Sand, 2013). Fatores interferiram negativamente no aleitamento } \\
\text { materno na primeira hora (AMPH) (Sá et al, 2016). }\end{array}$ \\
\hline $\begin{array}{c}\text { Papel dos profissionais } \\
\text { no ciclo gravídico } \\
\text { puerperal }\end{array}$ & $\begin{array}{l}\text { Enfermeiro como facilitador e motivador (Tamara et al, 2017). Falta sensibilidade dos } \\
\text { profissionais enfermagem quanto demandas aleitamento materno. (Tamara et al, } \\
\text { 2017).Médica atende intercorrências. (Corrêa et al, 2017). Atitude de rechaço da } \\
\text { médica (Corrêa et al, 2017). Falta de olhar para as necessidades da puérpera } \\
\text { (Corrêa et al, 2017). Conhecimento técnico da enfermeira foi considerado } \\
\text { insuficiente para a consulta puerperal (Corrêa et al, 2017). Foco no saber médico } \\
\text { (Corrêa et al, 2017).Manifestação de vulnerabilidade emocional na conversa com o } \\
\text { médico (Corrêa et al, 2017). Foco no exame obstétrico e não realização do exame } \\
\text { físico completo (Corrêa et al, 2017). }\end{array}$ \\
\hline
\end{tabular}


Temas

Critérios para avaliação

do cuidado no ciclo

gravídico-puerperal

\section{Resultados}

Avaliação do pré-natal, parto e puerpério (Hass, Teixeira \& Beghetto, 2013; Costa et al, 2013; Matozinhos et al, 2014; Andrade et al, 2015, Segatto et al, 2015; Queiroz, Soares \& Oliveira, 2015; Melo, Oliveira \& Mathias, 2015).

\section{DISCUSSÃO}

Nos estudos dessa Revisão Integrativa da Literatura (RIL), foram identificadas ações desenvolvidas pela equipe de saúde no ciclo gravídico puerperal. As ações de saúde nesse ciclo devem pautar-se principalmente nos aspectos relacionados à proteção e promoção em saúde. As atividades realizadas no acompanhamento pré-natal são descritas por Pedraza (2016) e Santos et al (2016), considerando algumas variáveis tais como de número de consultas por profissional, início do pré-natal, procedimentos de rotina, exames realizados e consulta puerperal. No âmbito da promoção da saúde, encontrou-se que as consultas são espaços de preparo para ser mãe (Demarchi et al, 2017), além de haver apoio da equipe para a amamentação (Barbosa et al, 2018). Observou-se como ação das equipes de saúde a realização de visita domiciliar no puerpério, mais especificamente da enfermeira e do Agente Comunitária de Saúde (ACS), não recebendo visita de outros profissionais da equipe (Corrêa et al, 2017). Ressalta-se ainda que as puérperas consideram as orientações do ACS como insuficientes (Corrêa et al, 2017). Além de ser relatada frustração por não haver consulta de enfermagem no pré-natal e puerpério (Demarchi et al, 2017). Neste sentido, ressalta-se a importância da interdisciplinaridade no cuidado à essas mulheres, visto que é atribuição da equipe de saúde o atendimento integral, respeitando-se a autonomia e saber de cada profissão. Peduzzi, Agreli, Silva \& Souza (2020) trazem a compreensão de trabalho em equipe interprofissional como um trabalho coletivo que se estabelece na relação recíproca entre as intervenções técnicas e as interações dos agentes envolvidos. As puérperas apontam que as suas necessidades não são atendidas (Corrêa et al, 2017), visto que não há programação de consultas às mesmas, sendo que a criança é o foco de atendimento no puerpério, inclusive a abordagem de anticoncepção realizada na consulta de puericultura (Corrêa et al, 2017). Os cuidados no puerpério devem ser individualizados, a fim de atender às necessidades da mãe e do bebê. Além da visita domiciliar, é preconizado o retorno da mulher e do recém-nascido ao serviço de saúde para avaliação física e acolhimento das necessidades das famílias (Brasil, 2013). Ainda, a investigação, revelou que há uma expectativa de que a equipe investigue suas necessidades e esclareça dúvidas (Corrêa et al, 2017). Dentre as ações desenvolvidas pelas equipes de saúde no ciclo gravídico-puerperal, nessa investigação, destacou-se a educação em saúde. Essa é uma 
ferramenta potente para construção da autonomia dos sujeitos. Assim, identificou-se a abordagem de temas relacionados ao ciclo gravídico puerperal por um membro da equipe de saúde ( Guerreiro et al, 2014; Oliveira et al, 2015), assim como o trabalho realizado pela equipe de saúde (Barbosa et al, 2015) e aprendizagem por familiares e redes sociais (Guerreiro et al, 2014).

Valdes, Santos \& Prado (2017), apontam que ocorrem abordagens tanto de maneira individual quanto coletiva. Houve orientações sobre aleitamento materno, as quais foram realizadas pelo médico, e menos da metade das mulheres acompanhadas durante o prénatal receberam tais orientações. (Rodrigues et al, 2013). Outras ocorreram no período puerperal, e quase todas as mulheres apresentaram dificuldades no processo da amamentação e somente uma realizava aleitamento materno exclusivo. (Corrêa et al, 2017). Destacando que as orientações podem ser consideradas suficientes e insuficientes (Silva et al, 2014; Tamara et al, 2017). Quanto às orientações em grupo, foram sinalizadas como importantes (Monteiro e Oliveira et al, 2016), possibilitando sanar dúvidas sobre o aleitamento materno no pré-natal e puerpério, bem como o cuidado com a criança (aparecida et al, (2014). Em relação a importância de orientações, emergiu que primíparas têm mais necessidades que multíparas (Oliveira et al, 2015). Apesar das multíparas terem experiências anteriores, acredita-se que sua participação no trabalho em grupo é essencial para troca e construção de conhecimentos. Nesse sentido, o trabalho em grupo, como a possibilidade de reunir primíparas e multíparas, de diferentes faixas etárias e diversas idades gestacionais, oportuniza compartilhar experiências, sanar dúvidas e construir a promoção do autocuidado na gestação, no parto e no puerpério. (Lima; Hollanda; Oliveira; Santos \& Carvalho, 2019). Houve questionamento sobre as informações realizadas no prénatal focarem o papel de mãe, da saúde do bebê, em detrimento a saúde da mulher (Cabral, Hirt \& Sand, 2013). Além de as mulheres trazerem que a construção do conhecimento foi por meio de uma educação verticalizada tanto na escola como nos serviços de saúde (Guerreiro et al, 2014) o que não contribui para uma aprendizagem efetiva. O trabalho em equipe remete-se a como está organizado o processo de trabalho dos serviços de saúde. Nesse contexto, tem-se problemas identificados no agendamento, cujo serviço não garante consulta no período puerperal, sendo atendidas somente quando demandam (Corrêa et al, 2017). Por outro lado, existe um atendimento facilitado pela recepção para demanda de amamentação (Corrêa, 2017) e um destaque que o acesso ao agendamento é um facilitador do acompanhamento do pré-natal (Cabral, Hirt \& Sand, 2013). Alguns fatores relacionados 
ao cuidado prestado interferiram negativamente na amamentação na primeira hora (AMPH), como não ter realizado pré-natal de forma adequada, o parto cesáreo e mãe e filho não permanecerem em alojamento conjunto após o parto (Sá et al, 2016). Um estudo desenvolvido em Sergipe com o objetivo de analisar a qualidade do pré-natal a partir das recomendações do Programa de Humanização no Pré-Natal e Nascimento (PHPN), embora tenham atingido uma boa cobertura, indicaram alguns problemas, entre outros, o início tardio do pré-natal, o número insuficiente de consultas e poucas orientações referente ao momento que estão vivendo (Mendes et al, 2020). Dessa forma, a compreensão da necessidade do processo de trabalho ser subjacente às necessidades de saúde, oportuniza as equipes novos olhares, leituras mais abrangentes das necessidades, que podem se desdobrar em uma organização interprofissional ancorado na prática comunicativa entre a equipe e estes com os usuários e suas famílias (Peduzzi, Agreli, Silva \& Souza, 2020). Em relação a atuação dos profissionais no ciclo gravídico puerperal, evidenciou-se que o enfermeiro é considerado facilitador e motivador no cuidado em saúde (Tamara et al, 2017), porém destacou-se a falta de sensibilidade desses profissionais quanto às demandas do aleitamento materno (Tamara et al, 2017). A investigação explicitou as dificuldades dos profissionais trabalhar as necessidades de saúde da mulher nesse momento da sua vida , enfatizando o conhecimento técnico insuficiente da enfermeira para realização da consulta puerperal, pois há valorização do saber médico, no entanto, sinalizam atitude antiética do mesmo (Corrêa et al, 2017) e foco no exame obstétrico e não realização do exame físico visando a integralidade do cuidado (Oliveira et al, 2015). Entretanto, elas sinalizam, que no diálogo com o médico conseguem manifestar sua vulnerabilidade emocional (Corrêa et al, 2017). Neste estudo, destaca-se alguns critérios definidos para a avaliação do cuidado no ciclo gravídico-puerperal. Costa et al (2013), Hass, Teixeira \& Beghetto (2013), Matozinhos et al (2014) e Queiroz, Soares \& Oliveira (2015) analisaram o pré-natal, parto e puerpério de acordo com o Protocolo de Pré-Natal e Puerpério do Ministério da Saúde.

Hass, teixeira \& Beghetto (2013) e Queiroz, Soares \& Oliviera (2015) avaliaram número médio de consultas, início do pré-natal, procedimentos clínicos obstétricos com destaque para aferição de peso e pressão arterial e realização de exames laboratoriais. Costa et al (2013) analisaram os indicadores do início do pré-natal, o número de ultrassonografias e a participação em atividades educativas. Matozinhos et al (2014) analisaram a captação precoce das gestantes; desenvolvimento das seguintes atividades ou procedimentos durante a atenção pré-natal como exames laboratoriais, imunização antitetânica; avaliação 
do estado nutricional da gestante e do risco gestacional; prevenção e tratamento dos distúrbios nutricionais e registro em prontuário e cartão da gestante.

Alguns critérios foram utilizados para avaliar o cuidado pré-natal, como o início do atendimento versus idade gestacional, número mínimo de consultas, realização de exames laboratoriais e aferição e avaliação da altura uterina, pressão arterial sistêmica, peso e batimentos cardiofetais e apresentação fetal (Melo, oliveira \& mathias (2015).

Andrade et al (2015) analisam os hemogramas realizados durante o pré-natal; o ganho de peso; as intercorrências clínicas; a ingesta dos macronutrientes e micronutrientes; o uso de tabaco e álcool e a atividade física. Segatto et al (2015) analisam a porcentagem de gestantes que realizaram pré-natal, a primeira consulta no primeiro trimestre, no mínimo seis consultas de pré-natal, exames básicos, consulta de puerpério e cobertura vacinal da antitetânica.

É importante que a avaliação do pré-natal possa contemplar critérios mais amplos, com aspectos de infra-estrutura, processo de trabalho e resultado, permitindo constatar como está sendo a operacionalização do cuidado. Nesse sentido, identificou-se que uma pequena parcela de mulheres foram encaminhadas à maternidade, participaram de atividades de educação em saúde e realizaram os exames recomendados para o pré-natal e a quantidade de gestantes com início do pré-natal no primeiro trimestre e maior número de consultas, ficou abaixo do esperado; denotando que a equipe de saúde deve ampliar a captação das gestantes e a necessidade de ser efetivada a construção de políticas públicas voltadas para redução das iniquidades (Silva, Leite, Lima \& Osório, 2019).

\section{CONCLUSÕES}

Os resultados e discussão dos dados do presente estudo mostram que o objetivo de analisar a produção bibliográfica acerca da atenção à saúde da mulher no ciclo gravídicopuerperal foi contemplado. Considerando que as ações de saúde devem pautar-se principalmente nos aspectos relacionados à proteção e promoção em saúde, identificou-se fragilidades no atendimento às mulheres, em especial, as necessidades das puérperas. O resgate do trabalho interprofissional possibilita a construção da integralidade do cuidado, desenvolvendo ações que abordem a gestação, o parto e o puerpério em consulta individual e em grupo, considerando a mulher como protagonista durante todo processo. Em particular, no puerpério, a mulher, além de assumir o seu papel de mãe cuidando do recém- 
nascido, deve ser cuidada em suas necessidades de saúde. Constata-se que as unidades de saúde estão com dificuldades de operar na lógica da integralidade. Assim, ressalta-se a importância deste estudo para a investigação qualitativa aplicada à área da saúde durante o ciclo gravídico puerperal com a finalidade de melhorar a qualidade dos cuidados a serem prestados às mulheres. Identifica-se como limite da pesquisa o desenvolvimento da mesma com um número reduzido de artigos, no entanto, possibilitou uma reflexão de como as mulheres estão sendo cuidadas. Nesse sentido, evidencia-se a necessidade no investimento de políticas públicas no tocante ao atendimento das necessidades dessa população.

\section{REFERÊNCIAS}

BRASIL. Ministério da Saúde. Portaria ํo 1.459, de 24 de junho de 2011, Rede Cegonha no âmbito do SUS. Brasília, 2011.

BRASIL. Ministério da Saúde. Secretaria de Atenção à Saúde. Departamento de Ações Programáticas e Estratégicas. Coordenação-Geral de Saúde das Mulheres. Coordenação-Geral de Saúde da Criança e Aleitamento Materno. ApiceOn: Aprimoramento e Inovação no Cuidado e Ensino em Obstetrícia e Neonatologia. Brasília (DF): Ministério da Saúde, 2017.

Luz, Leandro Alves da, Aquino, Rosana, \& Medina, Maria Guadalupe. (2018). Avaliação da qualidade da Atenção Pré-Natal no Brasil. Saúde em Debate, 42(spe2), 111-126. https://doi.org/10.1590/0103-11042018s208

Spindola, T., Penna, L., Lapa, A., Cavalcanti, A., Silva, J., \& Santana, R. (2017). PERÍODO PÓS-PARTO NA ÓTICA DE MULHERES ATENDIDAS EM UM HOSPITAL UNIVERSITÁRIO. Enfermagem em Foco, 8(1), 42-46. doi:https://doi.org/10.21675/2357-707X.2017.v8.n1.847

Souza, M. T.; Silva, M. D.; Carvalho, R. Revisão integrativa: o que é e como fazer. Einstein, São Paulo, v. 8, n. 1, p. 102-106, 2010.

Ganong LH. Integrative reviews of nursing research. Res Nurs Health. 1987;10(1):1-11.

Whittemore R, Knafl K. The integrative review: updated methodology. J Adv Nurs. 2005;52(5):546-53.

Barbosa A.M et al. Percepcões maternas sobre a assistência nutricional no acompanhamento interdisciplinar do pré-natal e puerpério. Tempus, actas de saúde colet, Brasília 2018.

Valdes I.N.F, Santos E.O, Prado E.V Programa mais médicos: qualificação da atenção ao Pré-Natal e Puerpério na âmbito da Estratégia da Saúde da Família. Rev. APS 2017

Demarchi R.F et al. Percepção de gestantes e puérperas primíparas sobre maternidade. Rev enferm UEPE on line, Recife, 2017.

Corrêa M.S.M et al. Acolhimento no cuidado à saúde da Mulher no Puerpério. Cad. Saúde Pública, 2017.

Pedraza D.F Assistência ao pré-natal, parto e pós-parto no município de Campina Grande, Paraíba. Cad. Saúde Coletiva, 2016, Rio de Janeiro.

Santos S.M.P dos et al. Acessibilidade ao serviço de saúde da mulher no ciclo gravídico-puerperal. Cogitare Enferm, 2016.

Tamara L. de B et al. Apoio recebido por mães adolescentes no processo de aleitamento materno. Rev Enferm UEPE on line, Recife, 2017.

Rodrigues A.P et al. Caracterização sociodemográfica e obstétrica de puérperas internadas em alojamento conjunto no Brasil. Ciência y Enfermeria XXII, 2016.

Monteiro e Oliveira L.F et al. Vivência de puérperas adolescentes quanto à gravidez e trabalho de parto. Rev Enferm UFPE on line, Recife, 2016. 
Barbosa L.N et al. Prevalência de práticas educativas acerca do aleitamento materno exclusivo (AME) em Cuiabá - MT. Escola Anna Nery Revista de Enfermagem, 2015.

Oliveira J.C de $S$ et al. Assistência pré-natal realizada por enfermeiros: o olhar da puérpera. Revista de Enfermagem do Centro Oeste Mineiro, 2015.

Aparecida K. R. M da et al. Percepção das mães em relação ao aleitamento materno no período do pós parto. ABCS Health Sci, 2014.

Silva N.M da et al. Conhecimento de puérperas sobre amamentação exclusiva. Revista Brasileira de Enfermagem, 2014.

Guerreiro E.M et al. Educação em saúde no ciclo gravídico puerperal: sentidos atribuídos por puérperas. Revista Brasileira de Enfermagem, 2014.

Rodrigues D.P et al. Representações sociais de mulheres sobre gravidez, puerpério e ações educativas. OBJN, 2013.

Cabral F.B, Hirt L.M, Sand I.C.P.V de. Atendimento pré-natal na ótica de puérperas: da medicalização à fragmentação do cuidado. Rev Esc Enferm USP, 2013

Sá N.N.B de et al. Fatores ligados aos serviços de saúde determinam o aleitamento materno na primeira hora de vida no Distrito Federal, Brasil, 2011. Rev Bras Epidemiol, 2016.

Hass C.N, Teixeira L.B, Beghetto M.G. Adequabilidade da assistência pré-natal em uma Estratégia de Saúde da Família de Porto Alegre - RS. Rev Gaúcha Enferm. 2013

Costa C.S.C et al. Características do atendimento pré-natal na Rede Básica de Saúde. Rev Eletr Enf, 2013.

Matozinhos F.P et al. Avaliação da atenção pré-natal: estudo de base populacional. Rev APS, 2014.

Andrade B.D et al. Fatores nutricionais e sociais de importância para o resultado da gestação, em mulheres em acompanhamento na rede de atenção primária de Juiz de Fora. Rev Med Minas Gerais, 2015.

Segatto M.J et al Avaliação da assistência pré-natal em município do Sul do Brasil. Rev Enferm UFPI, 2015.

Queiroz D.J.M, Soares D.B, Oliveira K.C.A.N de. Avaliação da assistência pré-natal: relevância dos exames laboratoriais. Revista Brasileira Promoção Saúde, Fortaleza, 2015.

Melo E.C, Oliveira R.R de, Mathias T.A.de F. Fatores associados à qualidade do pré-natal: uma abordagem ao nascimento prematuro. Rev Esc Enferm USP, 2015.

Marina Peduzzi, Heloise Lima Fernandes Agreli), Jaqueline Alcântara Marcelino da Silva, Helton Saragor de Souza Trab. Educ. Saúde, 18(s1):e0024678; Rio de Janeiro, 2020.

Brasil. Ministério da Saúde. Secretaria de Atenção à Saúde. Departamento de Atenção Básica. Atenção ao prénatal de baixo risco [recurso eletrônico] / Ministério da Saúde. Secretaria de Atenção à Saúde. Departamento de Atenção Básica. - 1. ed. rev. - Brasília : Editora do Ministério da Saúde, 2013. 318 p.: il. - (Cadernos de Atenção Básica, n 32).

da Silva Lima, V., Silva Esteves de Hollanda, G., Morais de Oliveira, B., Gomes de Oliveira, I., Vieira Freitas dos Santos, L., \& de Lima Carvalho, C. (2019). Health education for pregnant women: the search for maternal empowerment over the puerperal-pregnancy cycle / Educação em saúde para gestantes: a busca pelo empoderamento materno no ciclo gravídico-puerperal. Revista de Pesquisa: Cuidado é Fundamental Online, 11(4), 968-975. doi:http://dx.doi.org/10.9789/2175-5361.rpcfo.v11.6822

Mendes, Rosemar Barbosa, Santos, José Marcos de Jesus, Prado, Daniela Siqueira, Gurgel, Rosana Queiroz, Bezerra, Felipa Daiana, \& Gurgel, Ricardo Queiroz. (2020). Avaliação da qualidade do pré-natal a partir das recomendações do Programa de Humanização no Pré-natal e Nascimento. Ciência \& Saúde Coletiva, 25(3), 793-804. Epub March 06, 2020.https://doi.org/10.1590/1413-81232020253.13182018

Silva EP, Leite AFB, Lima RT, Osório MM. Avaliação do pré-natal na atenção primária no Nordeste do Brasil: fatores associados à sua adequação. Rev Saude Publica. 2019;53:43. 